\title{
Social Prescribing: community-based referral in public health
}

What is social prescribing?

Social prescribing is a term used with increasing frequency to describe the process of enabling healthcare professionals to refer patients to a link worker, to co-design a non-clinical social prescription to improve their health and wellbeing (Social Prescribing Network, 2016). Original descriptions of social prescribing involved three components to a scheme; a referral from a healthcare professional, a consultation with a link worker and an agreed referral into the third sector (Friedlie and Watson, 2004; South 2008; Brandling and House 2009).

Social prescribing has developed to incorporate referrals into the Arts, the natural environment, reading on prescription schemes and schemes to support health behaviour changes especially for long-term conditions. Other social prescribing schemes work to develop community cohesion such as timebanking, or address the social determinants of health by providing support for welfare, debt advice, housing and employment. In recent years social prescribing has emerged as a strategy for tackling health inequities through partnerships between primary care and third sector organisations, and augmenting standard health and social care services. In practice, social prescribing provides an architecture to allow a person to move between professional sectors into their community; this was recently recognised in the General Practice Forward View (2016) which listed social prescribing as one of the ten high impact actions. Social prescribing is, therefore, able to address physiological, physical, psychological, psychosocial, or socioeconomic issues, as well as enhancing community wellbeing and social inclusion.

How can people benefit from social prescribing?

Recent reviews of social prescribing evaluations and research indicate multiple benefits reported by participants and referrers directly engaged in social prescribing, including: increases in self-esteem and confidence; improvements in psychological or mental wellbeing; reduction in anxiety and depression; improvements in physical health and lifestyle; reduction in GP visits and referrals; reduction in social isolation and loneliness; learning and acquisition of new skills (see Chatterjee et al 2017). Arts on Prescription, one of the earliest models of social prescribing, was established in the mid-1990s and was developed as a practice involving creative art workshops to support patients with anxiety and other mental health challenges [See Box 1]. In a national study, Hacking et al. (2008) evaluated the impact of arts activities for patients with common mental health conditions. Findings showed that arts programmes made participants feel more empowered and confident and reduced feelings of social exclusion and isolation. Where social prescribing schemes are working well, they are highly acceptable to health professionals but the evidence for social prescribing has 
typically been based on small scale, qualitative or mixed methods studies with low sample sizes, and only a small number of studies have suitable controls.

Reviews of social prescribing

In a review of 86 social prescribing schemes covering arts, exercise, education, green gyms, signposting, timebanks and Healthy Living Initiatives, more than half (53\%) had no published evaluation, whereas just under half contained evaluation of primary research material (Chatterjee et al 2017). Of those schemes with evaluation ( $n=40), 17$ employed quantitative methods which included eight randomised controlled trials, 16 employed qualitative methods and seven employed mixed methods. In another review of 15 evaluations of social prescribing programmes, most were reported as being limited by poor design and rated as a having a high risk of bias (Bickerdike et al 2017). In a review of the evidence of social prescribing to reduce the demand for health services and its cost implications, evidence revealed considerable reduction in demand on primary and secondary care, but concluded that the quality of evidence is weak, and without further evaluation, it is be premature to conclude that a proof of concept for demand reduction had been established (Polley et al 2017). Similarly, the evidence that social prescribing delivers cost savings is highly encouraging but requires further evidence.

Social prescribing has started in a very organic manner across the UK, to address the need to provide something other than, or additional to, prescription medication. Whist many studies into the effectiveness of social prescribing have methodological shortcomings, most evaluations report positive outcomes. Notwithstanding challenges with the evidence base for social prescribing, the practice continues to grow. The Social Prescribing Network, which is a network of over a thousand professionals engaged in social prescribing, have now released guidance for commissioners and health professionals on how to set up and commission successful social prescribing schemes (Polley et al 2017b). In addition to social prescribing being listed in the GP Forward View, NHS England have appointed a National Clinical Social Prescribing Champion for NHS England and at least half of all clinical commissioning groups are engaged in social prescribing. Moreover the Creative Health inquiry report from the All Party Parliamentary Group on Arts, Health and Wellbeing recommends that the Social Prescribing Network and NHS England support a greater amount of arts on prescription to be commissioned and incorporated into redesign of care pathways. Taken together these significant steps exemplify the appetite to consider social prescribing as part of an integrated approach to public health.

Box 1. Case Study: Arts and Minds is an arts and mental health charity, established in 2007 and based in Cambridgeshire. The charity is linked to Cambridgeshire and Peterborough Foundation NHS 
Trust and the former Cambridgeshire PCT. The charity offers a 12-week programme of two-hour art workshops for people experiencing mild to moderate depression and anxiety as an alternative to CBT. GPs, health promotion workers, occupational therapists, social workers, psychologists and counsellors can refer clients directly to Arts on Prescription using a referral form. Led by a professional artist and supported by a counsellor, each session offers the chance to work in various media (e.g. drawing, collage, clay and wirework) with the objective of decreasing anxiety and/or depression, while increasing wellbeing. The programme also includes facilitated group visits to museums and galleries.

\section{Authors:}

Helen Chatterjee, Division of Biosciences, University College London, 507B, Darwin Building, Gower Street, London WC1E 6BT, UK Email: h.chatterjee@ucl.ac.uk

Marie Polley, Department of Life Sciences, University of Westminster, London, UK; Co-Chair, Social Prescribing Network, University of Westminster, London, UK

Gavin Clayton, Executive Director, Arts and Minds, Cambridge, UK

Corresponding author: Helen Chatterjee, as above

\section{References:}

Bickerdike L, Booth A, Wilson PM, et al. 2017. Social prescribing: less rhetoric and more reality. A systematic review of the evidence. BMJ Open. DOI:10.1136/bmjopen-2016-013384.

Brandling J and House W (2009). Social Prescribing in general practice: adding meaning to medicine. British Journal of General Practice. 59(563) 454-456.

Chatterjee, HJ, Camic, PM, Lockyer, B \& Thomson, L.J. 2017. Non-clinical community interventions: a systematised review of social prescribing schemes. Arts \& Health: An International Journal of Research, Policy and Practice. DOI: http://dx.doi.org/10.1080/17533015.2017.1334002

Friedli, L. and Watson, S. (2004) Social prescribing for mental health. Durham: Northern Centre for Mental Health.

Hacking, S., Secker, J., Spandler, H., Kent, L. \& Shenton, J. 2008. Evaluating the impact of participatory art projects for people with mental health needs. Health \& Social Care in the Community. 16(6), 638-648. DOI:10.1111/j.1365-2524.2008.00789.x.

NHS England (2016) General Practice Forward View. London. Available at: https://www.england.nhs.uk/gp/gpfv/

Polley, M, Bertotti, M, Kimberlee, R, Pilkington, K \& Refsum, C. 2017. A review of the evidence assessing impact of social prescribing on healthcare demand and cost implications. University of 
Westminster. Available at: https://www.westminster.ac.uk/patient-outcomes-in-health-researchgroup/projects/social-prescribing-network

Polley M, Fleming J, Anfilogoff T and Carpenter A. 2017. Making sense of social prescribing. Available at: Link to availability to be added shortly.

Social Prescribing Network. 2016. Inaugural National Social prescribing conference report. Available at: https://www.westminster.ac.uk/patient-outcomes-in-health-research-group/projects/socialprescribing-network

South J, Higgins TJ, Woodall J, White SM. 2008. Can social prescribing provide the missing link? Primary Health Care Residential Development. 9: 310-318. 